Historic, Archive Document

Do not assume content reflects current scientific knowledge, policies, or practices. 



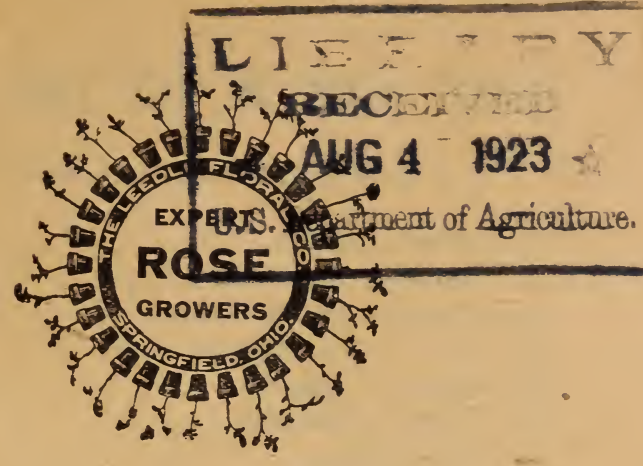

Bargains

11

in

Surplus Stock of

$T w 0=$ year $=01 d$
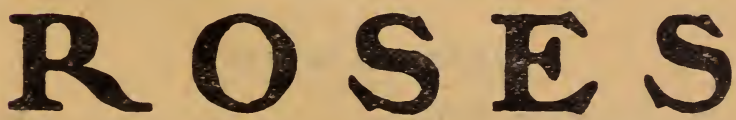

Semi=dormant, in 4=inch pots. Ship safely with all soil off. Splendid values for the prices. 


\section{Special prices for fall 1908 delivery}

\section{HYBRID PERPETUALS.}

Each.

10-Alfred Colomb.................. 7c

25 -Coquette des Alpes..............6. 6 c

12-Francois Levet.................. $6 \mathrm{c}$

30 -General Jacqueminot.............. 5c

15-Giant of Battles................ $5 \mathrm{c}$

12-Gloire Lyonnaise................. əс

50 -John Hopper................... इс

25 -La Reine...................... 5c

90 -Madame Masson................. $5 \mathrm{c}$

175 -Magna Charta.................... 5c

60 -Margaret Dickson................ ic

250 -Paul Neyron.................. $5 \mathrm{c}$

\section{Moss.}

$3 \bar{s}$-Henri Martin.................. 5 c

35-Madame de Rochambert........... 5c

70 -Mousseline $\ldots \ldots \ldots \ldots \ldots \ldots \ldots \ldots$.

30 -Princess Adelaide............... 5c

\section{DWARF RAMBLERS.}

125-Baby Crimson Ramblers...

(Mme. Norbert Levavasseur) ....... 5c

\section{HARDY CLIMBERS.}

35-Baltimore Belle.................. 5c

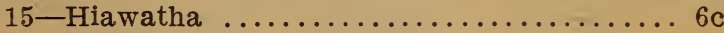

15-Pink Rambler................... 5c

75 -Prairie Queen................... 5c

25 -Seven Sisters................... 5c

18-Tennessee Belle................. 5c

22-English Sweet Briar.............. 5c 
12-Antoine Rivoire................ $7 \mathrm{c}$

90-Bessie Brown.................. 5 c

30 -Striped La France................ 5c

50 -White La France................. 万c

12-Mlle. Helene Gambier.............. 5c

BOURBON.

75 -Hermosa ..................... $5 \mathrm{c}$

3๊-Souv. de la Malmaison..............

\section{HYBRID POLYANTHA.}

12-Mosella (Yellow Soupert).......... 5c

\section{TEAS.}

20-Bougere ...................... 5c

20-Devoniensis .................... 5c

35-Madame de Wateville............... 5c

35 -Madame Lombard.................. 5c

12-Marie Guillot..................... 5c

25 -Rainbow $\ldots \ldots \ldots \ldots \ldots \ldots \ldots \ldots \ldots \ldots \ldots \ldots \ldots$

10 -The Queen................... 5c

\section{CLIMBING NOISETTES.}

215-Marechal Niel.................... \&c

50 -Mary Washington................ 5c

HYBRID NOISETTE.

18-Madame Wagram (Climbing Paul Neyron) 5c

\section{CLIMBING BENGAL.}

10-Empress of China................ 5c 


\section{Geton}

in the floral world by securing your share of the profit realizable from the purchase of bargains to be found only on

\section{the inside.}

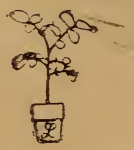

For complete assortment of $21 / 2$ and 4 -inch pot roses, see Fall 1908 Price List 